\title{
Molecular simulation of tracer and self- diffusion in entangled polymers
}

Article

Accepted Version

Shanbhag, S. and Wang, Z. (2020) Molecular simulation of tracer and self-diffusion in entangled polymers.

Macromolecules, 53 (12). pp. 4649-4658. ISSN 0024-9297 doi: https://doi.org/10.1021/acs.macromol.0c00680 Available at https://centaur.reading.ac.uk/90987/

It is advisable to refer to the publisher's version if you intend to cite from the work. See Guidance on citing.

To link to this article DOI: http://dx.doi.org/10.1021/acs.macromol.0c00680

Publisher: American Chemical Society

All outputs in CentAUR are protected by Intellectual Property Rights law, including copyright law. Copyright and IPR is retained by the creators or other copyright holders. Terms and conditions for use of this material are defined in the End User Agreement.

\section{www.reading.ac.uk/centaur}

\section{CentAUR}

Central Archive at the University of Reading

Reading's research outputs online 


\title{
Molecular Simulation of Tracer and Self-Diffusion in Entangled Polymers
}

\author{
Sachin Shanbhag*,† and Zuowei Wang ${ }^{*, \dagger}$ \\ †Department of Scientific Computing, Florida State University, Tallahassee, FL 32306 \\ $\ddagger$ Department of Mathematics and Statistics, University of Reading, Reading, RG6 6AX, UK \\ E-mail: sshanbhag@fsu.edu; zuowei.wang@reading.ac.uk \\ Phone: +91 850644 6548; +44 (0)118 3784618
}

TOC Graphic

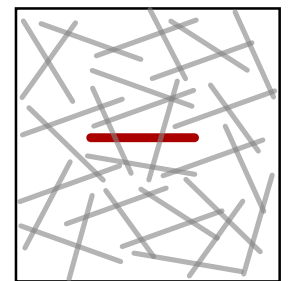

$D_{s}$

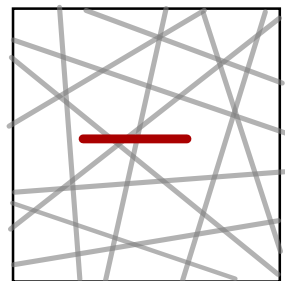

$D_{\infty}$ 


\begin{abstract}
The dependence of tracer diffusivity $\left(D_{\infty} \sim N^{-x_{\infty}}\right)$, where probe chains move in an environment of infinitely long matrix chains, and self-diffusion coefficient $\left(D_{s} \sim\right.$ $N^{-x_{s}}$ ), where probe and matrix chains are identical, on the molecular weight of the probe chain $N$ are investigated using three different molecular simulation methods, viz. molecular dynamics, the bond-fluctuation model (BFM) and the slip-spring (SS) model. Experiments indicate $x_{s} \approx 2.4 \pm 0.2$ over a wide intermediate molecular weight range, and $x_{\infty} \approx 2.0 \pm 0.1$, although the lower molecular weight limit for observing pure reptation in short probes is unclear. These results are partly inconsistent with some tube theories, and older, somewhat underpowered, molecular simulations. Estimating $x_{\infty}$ using brute-force BFM simulations is difficult because it involves large simulation boxes and long trajectories. To overcome this obstacle, an efficient method to estimate $D_{\infty}$ in which ends of matrix chains are immobilized, is presented and validated. BFM simulations carried out on systems with different probe and matrix chain lengths reveal that $x_{s}=2.43 \pm 0.07$, and $x_{\infty}=2.24 \pm 0.03$. Over a wider range of molecular weights, probe diffusivities obtained from the more coarse-grained SS model, calibrated with bead-spring molecular dynamics, reveal $x_{s}>x_{\infty}$, and $x_{\infty}>2$ for weakly and intermediately entangled chains. Tracer diffusivities obtained by artificially switching off constraint release in the SS simulations essentially overlap with probe diffusivities, strongly suggesting that constraint release is primarily responsible for the difference between $x_{s}$ and $x_{\infty}$. Nevertheless, both BFM and SS simulations indicate that below a certain chain length threshold, contributions of contour length fluctuations to $D_{s}$ and $D_{\infty}$ are important, and result in deviations from pure reptation scaling.
\end{abstract}




\section{Introduction}

Imagine a single probe polymer with $N_{p}$ monomers, immersed in a sea of matrix polymers with $N_{m}$ monomers each. The probe and matrix polymers are both long enough so that the average number of entanglement strands $Z_{p}=N_{p} / N_{e}$ and $Z_{m}=N_{m} / N_{e}$, where $N_{e}$ is the average number of monomers in an entanglement strand, exceed one. At fixed density and temperature, the diffusivity of the probe $D$ depends on both $Z_{p}$

and $Z_{m}$. Green and Kramer studied such systems using forward-recoil spectrometry, ${ }^{1}$ and fit experimental data on polystyrene melts to two prevailing theories for probe diffusion. ${ }^{2,3}$ The first theory modeled probe diffusion as a competition between reptation and constraint release $(\mathrm{CR}),{ }^{2}$ for which they found,

$$
D^{\mathrm{CR}}\left(Z_{p}, Z_{m}\right)=D_{\infty}\left(Z_{p}\right)\left(1+10.95 \frac{Z_{p}}{Z_{m}^{3}}\right)
$$

They were unable to discriminate between this theory and a competing theory by Klein, based on correlation between CR events, ${ }^{3}$ to which they could fit,

$$
D^{\mathrm{K}}\left(Z_{p}, Z_{m}\right)=D_{\infty}\left(Z_{p}\right)\left(1+5.45 \frac{Z_{p}}{Z_{m}^{2.5}}\right)
$$

In eqns. 1 and 2, the second term inside the parenthesis accounts for the acceleration in the diffusivity of the probe due to the finite size of matrix chains. To a first approximation, it is derived as the Rouse diffusivity of the long chain where the drag experienced by the beads is proportional to the reptation time of the short matrix chains. Regardless of the veracity of the underlying theories (both ignore contour length fluctuations or CLF), eqns 1 and 2 provide a useful mathematical structure to qualitatively describe probe diffusivity. Figure 1 depicts probe diffusivity for $Z_{p}=10$ using these two theories. $D$ decreases as matrix chains get longer, asymptotically approaching a terminal plateau. In this work, this $Z_{m}$-independent quantity is called the tracer diffusivity, $D_{\infty}\left(Z_{p}\right)=D\left(Z_{p}, Z_{m} \rightarrow \infty\right)$. The label "tracer diffusivity" is exclusively reserved for this scenario, where the probe chains move in a sluggish environment of infinitely long 
chains (marked by the subscript in $D_{\infty}$ ).

Another point of interest on figure 1 is indicated by the star at $Z_{m}=Z_{p}$, where the melt is monodisperse. The diffusivity corresponding to this point is the self-diffusivity $D_{s}\left(Z_{p}\right)=D\left(Z_{p}, Z_{m}=Z_{p}\right)$. Both self-diffusivity and tracer diffusivity are special cases of probe diffusivity. As a consequence, unlike $D$, both $D_{s}$ and $D_{\infty}$ depend only on $Z_{p}$, and the subscript " $p$ " on $Z_{p}$ (or $N_{p}$ ) may be dropped, when there is no ambiguity.

From eqns 1 and 2, we can obtain relationships between $D_{s}$ and $D_{\infty}$ :

$$
\left(\frac{D_{\infty}}{D_{s}}\right)^{\mathrm{CR}}=\frac{1}{1+10.95 Z_{p}^{-2}}, \quad\left(\frac{D_{\infty}}{D_{s}}\right)^{\mathrm{K}}=\frac{1}{1+5.45 Z_{p}^{-1.5}}
$$

According to these expressions, $D_{\infty}$ is smaller than $D_{s}$; however, as $Z_{p}$ increases, the difference between the two shrinks, and the ratio $D_{\infty} / D_{s}$ approaches one. Thus, $D_{s} \approx D_{\infty}$ for probes that are sufficiently long, say $Z \gtrsim Z_{1}^{*}$, where $Z_{1}^{*}$ is the "first" critical number of entanglements. Preliminary signs of this convergence can be observed in figure 1 even for $Z_{p}=10$, where $D_{\infty} / D_{s} \approx 0.85-0.90$.

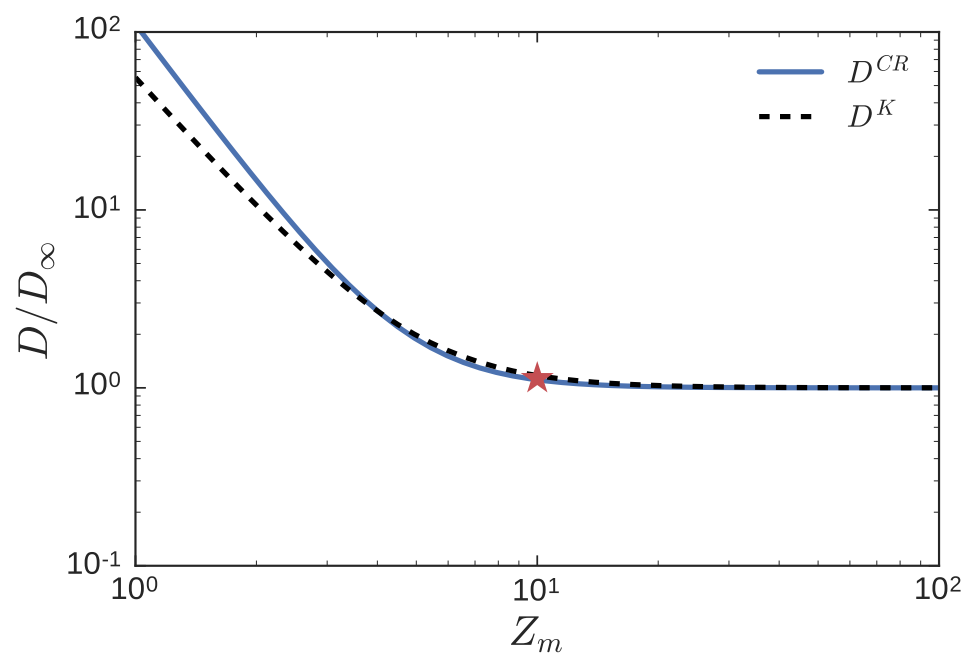

Figure 1: Normalized probe diffusivity for $Z_{p}=10$ using equations 1 (solid blue) and 2 (dashed black). Self-diffusivity $D_{s}$ is the probe diffusivity at $Z_{m}=Z_{p}$ (red star), while tracer diffusivity $D_{\infty}$ corresponds to the plateau at $Z_{m} \rightarrow \infty$. 


\subsection{Theory and Experiments}

Experimental studies on polymers of different chemistries, using various techniques are remarkably consistent with this framework. ${ }^{1,4-12}$ They find $D_{s} \sim Z^{-x_{s}}$ with $x_{s} \sim$ $2.4 \pm 0.2$ over a wide molecular weight range $\left(1<Z<Z_{1}^{*}\right),{ }^{10-12}$ and $D_{\infty} \sim Z^{-x_{\infty}}$ with $x_{\infty} \approx 2.0 \pm 0.1$ seemingly consistent with pure reptation for all chain lengths studied, as compiled in a meta-study by Wang. ${ }^{12}$ While experiments are more or less unanimous in a qualitative sense, some disagreement on the exact value of $x_{s}$ persists. It is difficult to resolve this issue conclusively due to the scatter in the data. This disagreement affects estimates of $Z_{1}^{*}$, where the crossover to the $D_{s} \approx D_{\infty}$ regime occurs. Taking $x_{\infty} \approx 2.0$, a stronger $Z$-dependence of $D_{s}\left(x_{s} \sim 2.4\right)$ suggests $Z_{1}^{*} \approx 20,{ }^{12}$ while a weaker dependence $\left(x_{s}=2.28\right)$ implies $Z_{1}^{*} \approx 100 .^{10,13}$

The compiled experimental data have been used to attribute the observed deviation from pure reptation $\left(x_{s}>x_{\infty}=2\right)$ to multi-chain or CR effects, rather than to singlechain or CLF effect. ${ }^{12,14} \mathrm{CLF}$ are active in both $D_{s}$ and $D_{\infty}$ measurements, while the effects of $\mathrm{CR}$ are suppressed in $D_{\infty}$ because $Z_{m} \gg Z_{p}$. Therefore, it is argued, any difference between $x_{s}$ and $x_{\infty}$ must originate from multi-chain CR effects.

Tube-based theories and simulations are somewhat at odds with experiments, and contend that the role of CLF is not fully acknowledged in the argument above. ${ }^{15-17}$ The picture they paint is richer, and is represented schematically in figure 2. For $Z \leq Z_{1}^{*}$, all the three modes of relaxation (reptation, CLF, and CR) are active in self-diffusion measurements, and $\mathrm{CR}$ is indeed responsible for $x_{s}>x_{\infty}$. However, unlike experiments, $D_{s}$ does not immediately transition to a pure reptation scaling $\left(D_{s} \sim Z^{-2}\right)$ for $Z>Z_{1}^{*}$. Instead, there is a broad intermediate regime $Z_{1}^{*} \leq Z \leq Z_{2}^{*}$, marked by the "second" critical entanglement number $Z_{2}^{*}$, in which $D_{s} \approx D_{\infty} \sim Z^{-2.25}$ has not fully transitioned to the pure reptation limit. ${ }^{13}$ Frischknecht and Milner found that their model indicates $Z_{1}^{*} \approx 20$ and $Z_{2}^{*} \approx 200-1000$. For $Z>Z_{2}^{*}$, the impact of CLF is weakened, reptation becomes the dominant mode of relaxation, and $D_{s} \sim Z^{-2}$ scaling is recovered. 


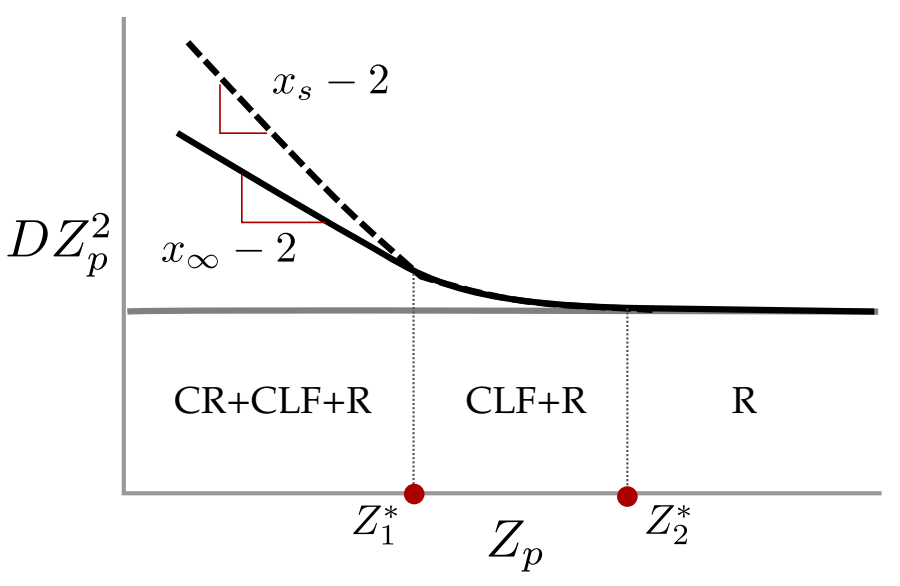

Figure 2: Scaled tracer (solid black line) and self-diffusion coefficients (dashed black line) with slopes of $x_{\infty}$ and $x_{s}$ for $Z_{p}<Z_{1}^{*}$. As $Z_{p}$ increases beyond a second threshold $\left(Z_{p}>Z_{2}^{*}\right)$, pure reptation scaling $D \sim Z_{p}^{-2}$ is recovered.

In the intermediate regime $\left(Z_{1}^{*}<Z<Z_{2}^{*}\right)$, switching $\mathrm{CR}$ on or off has negligible effect on the self-diffusivity in this model. ${ }^{13} \mathrm{~A}$ similar trend, $x_{s} \sim 2.4 \pm 0.1$ and insensitivity to CR, is also observed in the slip-link model simulations of Nair and Schieber for $Z>15 .{ }^{17}$ However, CLF effects persist, and are mainly responsible for $x_{s}>2$, which is consistent with experiments within reported uncertainty levels.

To summarize, there are two important points of difference between theory and experiments, which stem from different interpretations of the role of CLF. First, according to experiments $Z_{1}^{*} \approx Z_{2}^{*}$, and there is no intermediate regime where CLF effects endure. Second, for $Z<Z_{1}^{*}$ the numerical value of $x_{\infty}=2.25$ from the theoretical model ${ }^{13}$ differs from the pure reptation behavior of $x_{\infty}=2$ inferred from experimental data. $^{12}$

\subsection{Molecular Simulations}

Molecular simulations could help to unravel the differences between experiments and theory. Unfortunately, at the time Frischknecht and Milner proposed their explanation, ${ }^{13}$ molecular simulations were hardware-limited, and inconclusive. Indeed, studies available at that time showed a weaker dependence $x_{s} \approx 2.0-2.25$ for self-diffusion, ${ }^{18-22}$ and a stronger dependence $x_{\infty} \approx 2.3-2.5$ for tracer diffusion. ${ }^{23-26}$ Thus, molecular 
simulations at that time were inconsistent with both theory and experiments.

Since then, gains in computer speed have clarified the picture somewhat. Most previous results on $D_{s}$ that suggested $x_{s} \sim 2.0$ suffered from system sizes that were too small to avoid the influence of periodic images, ${ }^{27}$ insufficiently long simulations to fully resolve the diffusive regime, ${ }^{20}$ slopes inferred from only a few different values of $Z,{ }^{18}$ or neglect of corrections to pure reptation. ${ }^{21}$ The transition from the Rouse regime to a stronger power law $x_{s} \approx 2.4$ is sometimes reported for barely entangled chains $(1 \leq Z \leq 3.2) ;{ }^{28}$ however, other studies on chains of comparable length report a weaker $x_{s} \approx 2.1$ dependence, ${ }^{29,30}$ suggesting a possible transition to $x_{s} \approx 2.4$ at higher molecular weights. When these shortcomings are addressed, most subsequent molecular simulations using identical or comparable methods unambiguously yield $x_{s} \sim 2.4 \pm 0.2$ for moderately entangled polymers $(Z \approx 5-15) .{ }^{31-36}$

Likewise, studies that claimed $x_{\infty}$ closer to 2.4 instead of 2.0 , were based on relatively crude models (Evans-Edwards or repton) of single chains in a (typically cubic) obstacle network. ${ }^{23-26}$ Unlike experiments of tracer diffusion where the matrix is locally mobile but globally immobile, these calculations assumed that the matrix was fixed. Thus, the ability to draw strong inferences from these oversimplified molecular models is inherently limited. Ideally, a single molecular simulation technique is simultaneously used to study both $D_{s}$ and $D_{\infty}$.

While numerous studies of $D_{s}$ have been carried out, studying $D_{\infty}$ using simulations of bidisperse blends is challenging. First, matrix chains have to be sufficiently large to access the terminal plateau in figure 1 to estimate $D_{\infty}$. As $Z_{m}$ becomes large, the size of the simulation box required to avoid artifacts of periodic boundary conditions also increases. ${ }^{32}$ Furthermore, since a single or a small number of probe molecules are included in a simulation, the signal to noise ratio is compromised, and extremely long trajectories are required to resolve the diffusivity. Thus, simulations designed to study $D_{\infty}$ require large system sizes and long trajectories, both of which add to computational cost. 
Not surprisingly, most previous molecular simulations of bidisperse polymers involve at least one species that is unentangled or barely entangled. ${ }^{37-39}$ Picu and Raskhit considered a coarse-grained model of entangled bidisperse melts, where $Z_{p} \geq 5$. ${ }^{40}$ They observed that in binary blends, the diffusivity of the short chains was retarded, while that of the long chains was accelerated. They were able to observe the terminal plateau seen in fig. 1, where the diffusivity of the probe chain becomes independent of $Z_{m}$. However, the weight fraction of the "probe" chains in their calculations was greater than 0.6, and the extreme level of coarse-graining (40 LJ beads lumped into a blob) resulted in soft potentials for which chain uncrossability could not be guaranteed. Wang and Larson also performed molecular dynamics simulations of bidisperse polymers using a semi-flexible bead-spring chain model where both species were well-entangled $\left(Z_{p} \approx\right.$ 15). ${ }^{41}$ Low concentration of the probe chains ensured that entanglements between probe chains were nearly negligible. Their study did not investigate the $Z_{m}>Z_{p}$ regime, nor directly address $x_{\infty}$. Nevertheless, their results challenged the somewhat oversimplified CR Rouse picture, which presumed that CR is dominated by a single time scale corresponding to the relaxation time of the matrix chains. Instead, they found evidence for a broad spectrum of CR timescales.

\subsection{Motivation and Scope}

Thus, for $Z_{p}<Z_{1}^{*}$, there are still unresolved disagreements between certain theories $\left(x_{s}>x_{\infty}>2\right),{ }^{13}$ experiments $\left(x_{s}>x_{\infty} \approx 2\right),{ }^{12}$ and molecular simulations $\left(x_{\infty}>x_{s}\right.$, in older work $\left.{ }^{23,25,26}\right)$. After accounting for more recent molecular simulations, there is broad agreement that $x_{s}=2.4 \pm 0.2$. Previous estimates of $x_{\infty}$ from molecular simulations were based on unreliable over-simplified single chain models. Unlike $x_{s}$, newer work on bidisperse blends has not directly addressed $x_{\infty}$, in part because such probe simulations are computationally expensive. This work attempts to fill that gap. Two

other questions which animate this work are: "is CR responsible for the experimentally observed difference between $x_{s}$ and $x_{\infty}$ ?" and "can CLF lead to deviation of $x_{\infty}$ from pure reptation behavior (i. e., $x_{\infty}>2$ )?" 
We use three different molecular simulation techniques which are described briefly in section 2. In increasing order of coarse-graining, these methods are molecular dynamics (MD) based on a bead-spring chain model, ${ }^{18}$ the bond-fluctuation model (BFM), and the single-chain slip-spring (SS) model. Obtaining $D_{\infty}$ from MD, while desirable, is prohibitively expensive for large probes. Therefore, the primary tools for investigating the central question of this work, estimating $x_{s}$ and $x_{\infty}$, are the BFM and SS models. The SS model is calibrated using MD, ${ }^{42}$ and subsequently employed to extrapolate to systems that are not accessible with MD. The largest probes studied in this paper use the SS model, since it is the most coarse-grained. Furthermore, it is possible to switch off $\mathrm{CR}$ in the SS model, ${ }^{33}$ which allows us to directly address the role of CR in tracer diffusion.

The paper is organized as follows: Section 2 provides a description of the simulation techniques used, including clamped matrix and no-CR simulations that are eventually used to estimate $x_{\infty}$. In section 3 , we discuss results starting with self-diffusivity. Approximations introduced in the BFM to track tracer diffusivity are then validated, followed by a comparison of self and tracer diffusivities. Finally, we analyze the relative contributions of CR and CLF in the context of figure 1 in light of these new simulations.

\section{Model and Methods}

We briefly describe the molecular simulation techniques (MD, BFM, and the SS model) used in this paper. Since all these methods have been used extensively to study polymers in the past, only important features are summarized. We then describe the different settings used with these models (fig. 3) to estimate $x_{s}$ and $x_{\infty}$. All three simulation models use beads to represent monomers on a polymer chain. MD is the most fine-grained of these simulations, followed by BFM, and then the SS model. One bead in the BFM is approximately equivalent to $2 \mathrm{MD}$ beads, while one bead in the SS model is equivalent to $10 \mathrm{MD}$ beads.

It is useful to clarify the two roles that MD plays in this work. First, MD data 
on the diffusivities of short probe chains $\left(N_{p}=50-200\right)$ diluted in long matrix chains $\left(N_{m}=1000\right)$ have not been reported before for the bead-spring chain model described below. Previous MD studies of Wang and Larson on binary blends focused on long probe chains in short chain matrices to investigate the enhancement of CR effects by reducing the matrix chain length. ${ }^{41}$ Here, we are interested in the suppression of CR effects by using matrix chains much longer than the probe chains. Second, MD simulation results are used to calibrate and validate the slip-spring model simulations. While this was previously done for monodipserse polymer melts, ${ }^{42,43}$ the validity of such mapping for the binary blends is reported here.

\subsection{Molecular Dynamics}

Polymers are represented by the standard Kremer-Grest bead-spring model. ${ }^{18}$ All monomers interact via purely repulsive Lennard-Jones (LJ) potential with the LJ parameter $\epsilon=1.0 k_{B} T$, where $k_{B}$ is Boltzmann's constant and $T$ is the absolute temperature, and a cutoff radius of $r_{c}=2^{1 / 6} \sigma$ where $\sigma$ is the bead diameter. Monomers on a chain are connected to their neighbors by a finitely extensible nonlinear elastic (FENE) potential with the spring constant $k=30 \epsilon / \sigma^{2}$ and the maximum bond length $R_{0}=1.5 \sigma$. Chains are flexible, and the bending potential is assumed to be zero, since such chains are more suitable for mimicking real polymers studied in experiments. ${ }^{42}$ The corresponding statistical segment length is $b=1.31 \sigma$. The number density of the bonds is set to a fixed value of $\rho=0.85 \sigma^{-3}$ for all simulated systems. The length and

time scales in the systems are set by $\sigma$ and $\tau_{L J}$, respectively. ${ }^{18,42,44,45}$ The number of beads per entanglement strand in such model melt systems has been reported to range from $N_{e}=35$ to 85 , depending on the observables and methods used for the analysis. ${ }^{18,42,46-51}$ For example, a value of $N_{e} \approx 50$ was found from the crossover behavior of the middle-monomer mean-squared displacements of monodisperse linear chains with $N=1000$ from the Rouse to entanglement regimes. ${ }^{42}$ The initial configuration of a melt is prepared using the equilibration method developed by Auhl et al. so as to start the simulations in close proximity to the equilibrium states. ${ }^{44,52}$ Trajectories are 
obtained by integrating Langevin equations of motion for the monomers, using the velocity Verlet algorithm with time step $\Delta t=0.012 \tau_{L J}$. Simulations are performed in the NVT ensemble with periodic boundary conditions applied along all three directions of the cubic simulation box. Unless specified, molecular dynamics (MD) results are presented in reduced units of $m=\sigma=\epsilon=1$.

\subsection{Bond-Fluctuation Model}

We use Shaffer's version of the bond-fluctuation model (BFM), which is a popular lattice Monte Carlo method to study polymer melts. ${ }^{20}$ Polymers are represented by monomers on a simple cubic lattice $(L \times L \times L)$. The length of bonds between monomers is restricted to the set $\{1, \sqrt{2}, \sqrt{3}\}$, resulting in a model that is more flexible $\left(c_{\infty}=\right.$ 1.17) and coarse-grained than the original BFM. ${ }^{53-55}$ Probe chains are represented by $n_{p}$ polymers with $N_{p}$ monomers each. They are immersed in a matrix of $n_{m}$ chains with $N_{m}$ monomers each. At a total lattice occupancy $\phi=\left(n_{p} N_{p}+n_{m} N_{m}\right) / L^{3}=0.50$, the BFM is well-suited to model polymer melts. ${ }^{20}$

At each trial, a random local displacement of a randomly selected monomer is attempted. It is rejected if the trial violates excluded volume (target site already occupied), chain uncrossability (intersection of bond midpoints), or finite extensibility (bond-lengths greater than $\sqrt{3}$ ). ${ }^{20}$ One Monte Carlo Step (MCS) involves $n_{p} N_{p}+n_{m} N_{m}$ such trials, so that each monomer is selected for displacement on average once per MCS. The units of length and time are lattice spacing and MCS. The BFM can be exploited for rapid initial equilibration. ${ }^{56}$

In the past, we have successfully used the BFM to study entanglements, ${ }^{54,56,57}$ diffusion in ring-linear blends, ${ }^{58,59}$ and probe diffusion. ${ }^{60,61}$ From this body of work, $N_{e} \approx 30$ using primitive path analysis, ${ }^{54}$ the average primitive path step length is approximately 8.6, and $\tau_{e} \approx 5000$ MCS. ${ }^{55}$ For monodipserse linear systems with $N$ monomers, the radius of gyration and self-diffusion coefficient are given by, $R_{g}^{2} \approx$ $0.41(N-1)$ and $D_{s} \approx 1.8 N^{-2.4}$, respectively. 
During the simulation, the location of the center-of-mass of the probe chains is tracked. From these trajectories, the mean-squared displacement (MSD) of the probe chains is computed. Weighted least squares with statistical bootstrap is used to infer the self-diffusivity and the associated uncertainty. ${ }^{60,62}$

\subsubsection{Clamped Matrix Chain Ends}

Consider the following question: "for $N_{p}=300$, how large should $N_{m}$ be so that the measured probe diffusivity $D\left(N_{p}, N_{m}\right)$ is within, say, $1 \%$ of $D_{\infty}$ ?" Eqn. 2, for example, suggests $N_{m} \approx 900$, which would require box sizes greater than $L \approx 80-90$ to avoid artifacts of periodic boundary conditions. The computational cost per MCS is proportional to the number of monomers, and increases as $L^{3}$. Most of this effort is expended on matrix chains that are not the subject of interest. If possible, it would therefore be advantageous to estimate $D_{\infty}$ using smaller simulation boxes.

As $N_{m} \rightarrow \infty$, matrix chains are locally mobile, but globally immobile, on the order of timescales corresponding to the diffusion of the probe chains. Such an effect can be recreated by clamping or immobilizing the ends of finite matrix chains, allowing us to estimate $D_{\infty}$ using smaller simulation boxes. We label these "short-cut" calculations as clamped matrix (CM) simulations. In CM simulations, monomers at the two ends of a matrix chain (monomers numbered 1 and $N_{m}$ ) are effectively clamped, rendering the chains "globally immobile". Nevertheless, internal portions of these chains are free to move within these constraints. No such constraint is applied to the probe chains; their ends remain mobile.

We also perform "regular" probe simulations (bidisperse blends) where the ends of the matrix chains are not clamped. Diffusivities extracted from CM simulations are decorated with a "hat" $(\hat{D})$ to distinguish them from regular simulations of bidisperse polymers $(D)$. Later, we show that $\hat{D}\left(N_{p}, N_{m}\right)$ is independent of $N_{m}$ (for $N_{m} \gtrsim$ $75-100)$, and provides a reasonable estimate of $D_{\infty}=D\left(N_{p}, N_{m} \rightarrow \infty\right)$ without resorting to large $N_{m}$ or $L$. 


\section{$2.3 \quad$ Slip-Spring Simulations}

The single-chain slip-spring model was initially developed by Likhtman for studying the entanglement dynamics of linear chains. ${ }^{33}$ The basic building block of this model is a Rouse chain of $N$ beads. Topological constraints due to entanglements are modeled by a set of virtual springs, each represented using $N_{s}$ beads. One end of each virtual spring is connected to a Rouse monomer by a slip-link, while the other end (anchor point) is fixed in space. On average, there is one slip-link per chain segment of $N_{e}^{S S}$ monomers. Following the revised version of the SS model, ${ }^{42,63,64}$ slip-links move along the chains discretely, by hopping from one Rouse bead to a neighboring bead with an acceptance rate controlled by a Metropolis Monte Carlo scheme. Slip-links on a given chain are not allowed to pass through one another, or cohabit the same monomer. Furthermore, each slip-link is paired with another slip-link sitting on a different chain.

The creation or destruction of slip-links involves at least one chain end. If a sliplink is deleted from the free end of one chain, its associated partner is simultaneously deleted. A new pair of coupled slip-links is created immediately, one at one free end of a randomly chosen chain and the other on an unoccupied monomer randomly selected on any other chain. Thus, the total number of slip-links in the system remains constant. Following previous work, ${ }^{42,43,48,63,64}$ SS model parameters are set to $N_{e}^{S S}=4$ and $N_{s}=0.5$. In previous simulations of monodisperse entangled linear chains, excellent agreement was obtained between the SS and MD models for the mean-squared displacement of the central monomers, when one Rouse bead in the SS model was mapped to 10 beads in the MD model. ${ }^{42}$ Simultaneously, the SS time had to be rescaled by a factor of $\tau_{S S}^{M D}=3370 \tau_{L J}$.

Due to this level of coarse graining, SS simulations can extrapolate MD predictions for high molecular weight polymers over long time periods. However, the advantage is not only computational, but also conceptual. For example, in the SS model CR can be switched on or off, and its effects can be examined. Note that this is not possible with BFM or MD, which makes the SS model uniquely useful for the current study. 


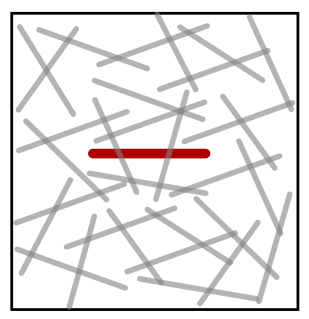

(a)

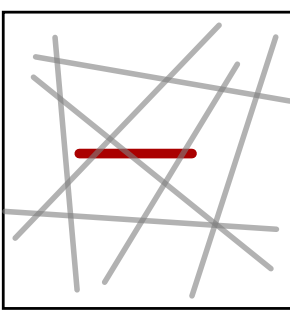

(b)

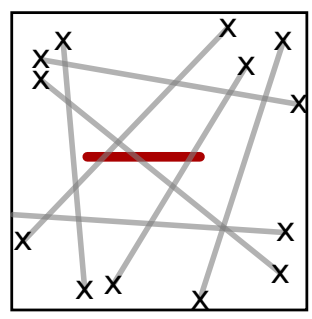

(c)

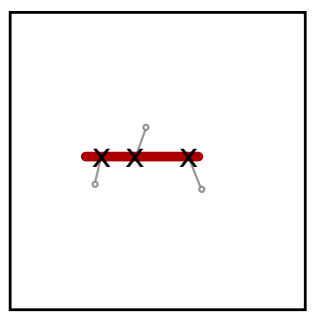

(d)

Figure 3: Schematic representation of different simulations. The thick red line denotes the probe chain and the gray lines denote matrix chains. In studies of (a) self-diffusion, the size of the probe and matrix chains is identical $\left(N_{p}=N_{m}\right)$, while in (b) probe and (c) tracer diffusion $N_{p} \neq N_{m}$, in general. In clamped matrix studies for tracer diffusion (c), the ends of the matrix chains are immobilized. In the SS model, CR can be turned off, which corresponds to diffusion in a fixed network $(\mathrm{d})$.

\subsection{Systems}

We perform four different types of calculations in this work, schematically shown in fig. 3. All three methods (MD, BFM, and SS) are used to study self- and probe diffusion shown in fig. 3(a) and 3(b). The BFM is used for CM simulations shown in fig. 3(c), while CR can be switched off only in the SS model as shown in fig. 3(d). The last two settings are proxies for tracer diffusion.

(a) Self-Diffusion: This is a special case of probe diffusion with $N_{m}=N_{p}=N$ (see fig. 3a). The melt is therefore monodisperse. New systems modeled in this work with MD, BFM, and SS are reported in Supporting Information (SI) Table 1. The longest chains modeled using SS correspond to $Z \approx 30$, based on the entanglement length $N_{e} \approx 50$ as estimated in MD simulations from the crossover behavior of chain middle-monomer MSD from Rouse to entanglement regimes. ${ }^{42}$

(b) Probe Diffusion: In these simulations, $N_{p}$ is not necessarily equal to $N_{m}$ (see fig. 3b). In BFM, the monomer number fraction of the probe chains is set to $f_{p}=n_{p} N_{p} /\left(n_{p} N_{p}+n_{m} N_{m}\right)=0.10$ to minimize interaction between probes. This corresponds to concentrations that are about four times smaller than the overlap concentration $\left(\approx 3.8 / \sqrt{N_{p}}\right)$, even for the longest probe chains $\left(N_{p}=300\right)$ considered. New simulations performed here are summarized in table 2 (SI). 
The binary blend systems studied using MD and SS model simulations are listed in table 3 (SI). In these binary blends, the matrix chains are all of length $N_{m}=1000$ or $Z_{m} \approx 20$. The probe chain lengths range from $N_{p}=50-200$ or $Z_{p} \approx 1-3$ for $\mathrm{MD}$, and $Z_{p} \approx 1-10$ for $\mathrm{SS}$, respectively. The monomer number fraction of the probe chains is fixed at 0.15 in order to get reasonably good statistics. This fraction is slightly higher than that typically used in tracer diffusion experiments, including the BFM simulations performed here. This does not appear to affect the overall conclusions, which is demonstrated by comparing SS simulations with and without CR.

(c) Clamped Matrix Simulations: These simulations are only performed with the BFM to estimate $D_{\infty}$ by clamping the ends of matrix chains (see fig. 3c). As in probe diffusion studies, $f_{p}=0.10$. These systems are identified in table 2 (SI) with an " $\mathrm{x}$ " in the last column. The diffusivities corresponding to these simulations are decorated with a hat $(\hat{D})$.

(d) No-CR Simulations: In the SS model, CR can be switched off to quantify the extent of the constraint release effect. In these simulations, the slip-links are not coupled with each other and so can only be deleted or added from the chain ends. Probe chains effectively move about in an environment similar to a permanent network by reptation and CLF. The diffusivities corresponding to these simulations are denoted by $\tilde{D}$.

Normalizing chain lengths with $N_{e}$ allows us to directly compare different simulation methods and experiments on different polymers. Here, we use $N_{e} \approx 30$ for the $\mathrm{BFM}$, and $N_{e} \approx 50$ for the flexible chains in the MD, and correspondingly SS calculations. However, it is acknowledged that there is no unique way of determining the entanglement molecular weight in either experiments or simulations. It, unfortunately, depends on the properties of interest, and the method of analysis. ${ }^{49,51,57}$ For example, Wang and Larson found considerable variation in the estimated $N_{e}$ for semi-flexible KG bead-spring chains using different dynamic observables and different regimes. ${ }^{41} \mathrm{~A}$ 
similar finding was reported by Harmandaris and Kremer on polystyrene melts where the $N_{e}$ values estimated from segmental dynamics $(110 \pm 30)$, primitive path analysis $(205 \pm 20)$ and self-diffusion coefficients (240-300) showed significant variation. ${ }^{29}$ For the flexible Kremer-Grest MD model used here different values of $N_{e}$ between $35-85$ have previouly been justified. ${ }^{18,42,46-51}$ Luckily, $x_{s}$ and $x_{\infty}$ are not sensitive to the precise value of $N_{e}$, and the conclusions of the paper are not significantly compromised by $15-20 \%$ variation in the value of $N_{e}$.

\section{Results and Discussion}

We first consider $D_{s}$ using the three simulation methods. Next, we consider probe and $\mathrm{CM}$ diffusion studies using the BFM and show how $D_{\infty}$ can be extracted from $\hat{D}$. Finally, we compare $D_{s}$ and $D_{\infty}$ obtained using CM simulations with the BFM and SS simulations without CR. The simulation results are then be used to assess and understand the discrepancy between the theoretical predictions ${ }^{13}$ and compiled experimental data ${ }^{12}$ on $D_{s}$ and $D_{\infty}$.

\subsection{Self-Diffusion}

Figure 4 plots the self-diffusivity as a function of the average number of entanglement strands per chain. For clarity, in all the SS model results reported henceforth, the number of beads or entanglements and the self-diffusion coefficients are mapped and reported in MD units. In the figure, diffusivities from MD and SS simulations are shifted downwards by a multiplicative factor of 0.3 , so that they can be directly compared with BFM results. Numerical values of the diffusion coefficients are reported in SI tables $4-7$.

BFM results are shown in figure 4 by circles. It includes previous data from Shanbhag's group and others, and also new simulations performed in this study to

increase the reliability of the inferred power-law. ${ }^{20,35,65,66}$ Shaffer's results are qualitatively consistent with the rest, although error-bars are not provided in his study. He 


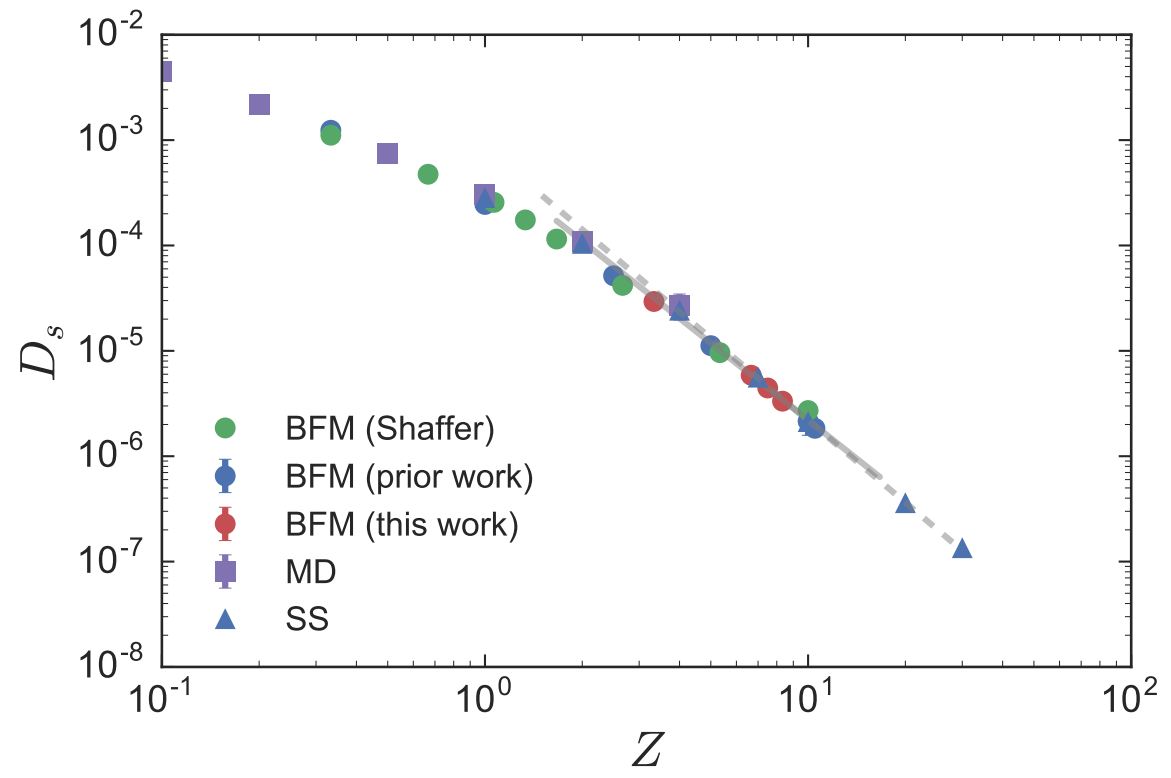

Figure 4: Self-diffusion coefficient as a function of the number of entanglements for the BFM (circles), MD (squares), and SS (triangles) simulations. Error bars are comparable with the size of the symbols. For the BFM, results from Shaffer (green circles), ${ }^{20}$ previous data points from Shanbhag's group (blue circles), and new simulations performed for this study (red circles) are shown. For $Z>3$, the best-fit line $D_{s}=5.84 \times 10^{-4} Z^{-2.43}$ is shown in gray. Diffusivities from MD and SS simulations are shifted by a factor of 0.3 for easier visualization. The best-fit regression through the SS data is $2.84 \times 10^{-3} Z^{-2.59}$, shown by the dashed gray line. 
used these $D_{s}$ (green circles) to infer $D_{s} \sim Z^{-2.09} \cdot{ }^{20}$ However, his analysis suffered from two significant shortcomings: (i) only three data-points with $Z \geq 2(N=80$, 160, and 300) were used, and (ii) system sizes were relatively small - both in terms of the number of chains in a box, and the length of a simulation run. The overestimation of $D_{s}$ visible in figure 4 at $Z=10$ is a classic signature of these shortcomings. Consequently, the single, potentially problematic, data point at $Z=10$ skews the value of $x_{s}$ extracted towards 2. In contrast, newer simulations reported in the figure use system sizes that are an order of magnitude larger, and are hence more reliable. Using these results on modestly entangled systems $3<Z<11$, we obtain the weighted least-squares fit $D_{s}=(5.84 \pm 0.78) \times 10^{-4} Z^{-2.43 \pm 0.07}$.

A nearly identical situation was previously reported with the original BFM, in which small system sizes, and too few data points were again used to incorrectly infer $x_{s}=2.0 .{ }^{27}$ When these shortcomings were fixed by the systematic replication studies of Hagita and Takano, ${ }^{32}$ the same model showed $x_{s}=2.44$ for $Z \approx 3.5-7$, in line with experiments, and the version of the BFM employed here.

The self-diffusivity obtained from MD simulations is consistent with previous studies using a flexible chain Kremer-Grest model over a comparable range of chain lengths. ${ }^{18,30}$ The shifted MD and SS simulations largely follow the trend of $D_{s}$ obtained from BFM simulations. For $Z>3$, SS simulations show a somewhat stronger dependence $D_{s} \sim Z^{-2.59 \pm 0.05}$ with chain length up to $Z \approx 30$. This is slightly larger than the $x_{s} \approx 2.4$ reported by Likhtman using the original version of the SS model. ${ }^{33}$ This quantitative difference may be related to the different effective frictions imposed by the slip-springs on the Rouse chains ${ }^{67}$ when changing the slip-link motion between adjacent beads from the original continuous mode ${ }^{33}$ to the updated discrete mode as used in the current work. Another factor could be the different numbers and ranges of data points used for the fitting as well as the magnitude of the error bars, considering that the updated version of the SS model and enhanced computational power allow for longer simulation runs and better statistics. The multi-chain slip-spring and multi-chain slip-link (primitive chain network) model simulations of Masubuchi and 
Uneyama $^{36}$ observed similar values of $x_{s}$ before tentative signs of transition to pure reptation-like diffusion $\left(D_{s} \sim Z^{-2}\right)$ emerged around $Z_{2}^{*} \approx 35-50$ (see SI figure 1 ). The experimental data on $D_{s}$ of certain types of polymers, such as h-polybutadiene (PBD), polystyrene and 1,4-PBD, also demonstrate a decaying power of $x_{s} \approx 2.5-2.6$ in the chain length range of $Z_{p} \lesssim 20-30 .{ }^{12}$

It should be pointed out that some amount of variation in $x_{s}$ is unavoidable because it is extracted from an intermediate molecular weight range $(Z=3-30$ for SS model, and $Z=3-11$ for BFM, in this work). The lower limit has to be sufficiently above $Z=1$ to ensure that chains are entangled, while the upper limit has to be below the expected cross-over point to reptation-like scaling. For $Z$ below the lower limit, the melt is unentangled, where $D_{s} \approx Z^{-1}$. For $Z$ above the upper limit, $D_{s} \approx Z^{-x_{\infty}}$. Thus, on both sides of this intermediate range, the dependence of $D_{s}$ on $Z$ is weaker than $D_{s} \sim Z^{-x_{s}}$. Smooth transitions between the three regimes suggests that minor variation around $x_{s} \sim 2.4$ is not surprising, for different models and molecular weight ranges.

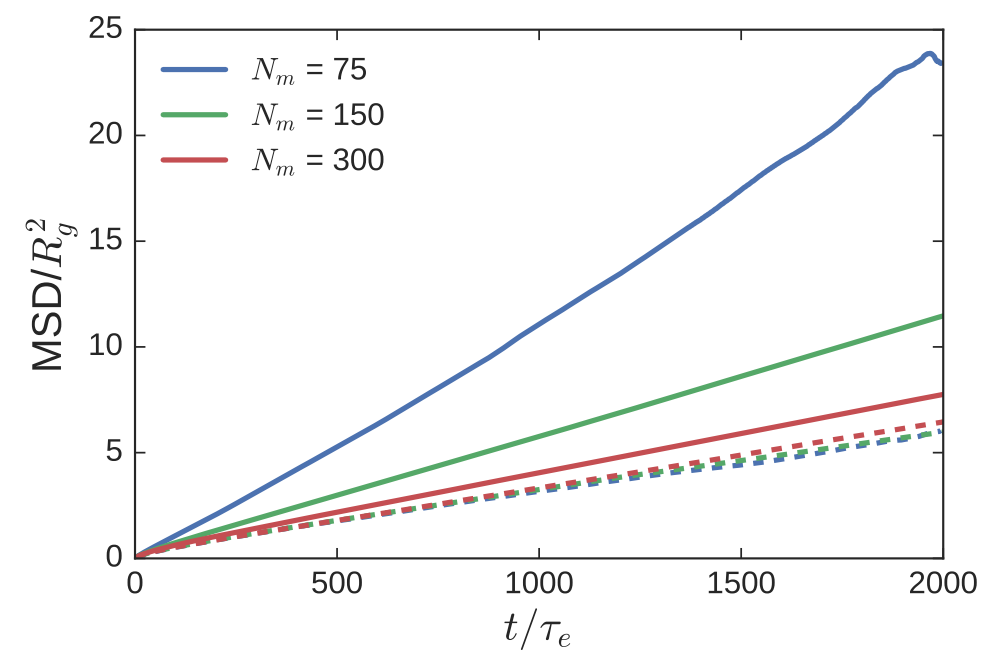

Figure 5: Center-of-mass mean-squared displacement of the $N_{p}=150$ probe, as the matrix chain length is varied, using the BFM. Dashed lines depict CM simulations, while solid lines of the same color depict probe simulations with identical $N_{m}$ and mobile matrix chain ends. 


\subsection{BFM: Probe and Clamped Matrix Diffusion}

The center-of-mass MSD $\left(g_{3}\right)$ of the $N_{p}=150$ probe chain using the BFM (corresponding to $Z_{p} \approx 5$ ), at three different matrix chain lengths, $N_{m}=75,150$, and 300 is reported in figure 5. Solid and dashed lines of a particular color show results from regular probe diffusivity (unfixed matrix chain ends), and CM simulations (fixed matrix chain ends), respectively. In regular simulations, probe chains are most mobile for $N_{m}=75$, and their mobility decreases as the length of the matrix chains increases to $N_{m}=300$. At large $t$, the slope of the MSD is proportional to $6 D$. In this example $\left(N_{p}=150\right)$, self-diffusivity $D_{s}$ corresponds to $N_{m}=150$ (solid green line). When matrix chains are longer $\left(N_{m}=300\right), D<D_{s}$, and vice versa. In contrast, the MSD curves from the CM simulations essentially overlap, and it is difficult to tell them apart. This implies that the diffusivities inferred from CM simulations, $\hat{D}$, are tightly clustered together. As $N_{m}$ increases, the MSD curves from regular simulations begin to approach the MSD curves from CM simulations.

These diffusivities for $N_{p}=150$ are plotted in figure 6 (green symbols), along with those for probe chains with $N_{p}=75$ (blue symbols), and 300 (red symbols). The size of the matrix chains is varied between $N_{m}=10-300$. To avoid confusion due to overlapping series, the diffusivities for $N_{p}=150$ and 300 , have all been shifted by a multiplicative factor of $1 / 5$ and $1 / 25$, respectively. The exact values of the diffusivities are tabulated in Supporting Information. For each $N_{p}$, probe diffusivity $D$ decreases as $N_{m}$ increases, approaching a plateau at large $N_{m}$. This is qualitatively similar to the trend shown in figure 1.

As $N_{p}$ increases, the $N_{m}$ required to approach the terminal region also increases. This can be seen in figure 6 . At $N_{m}=300, N_{p}=75$ appears to have comfortably settled into a plateau, whereas $N_{p}=150$ appears to have barely touched $D_{\infty}$. No sign of a plateau is visible for $N_{p}=300$.

The results of the CM simulations are shown by diamonds of the corresponding color. For $N_{p}=300$, only a single simulation run with $N_{m}=300$ was performed. For 


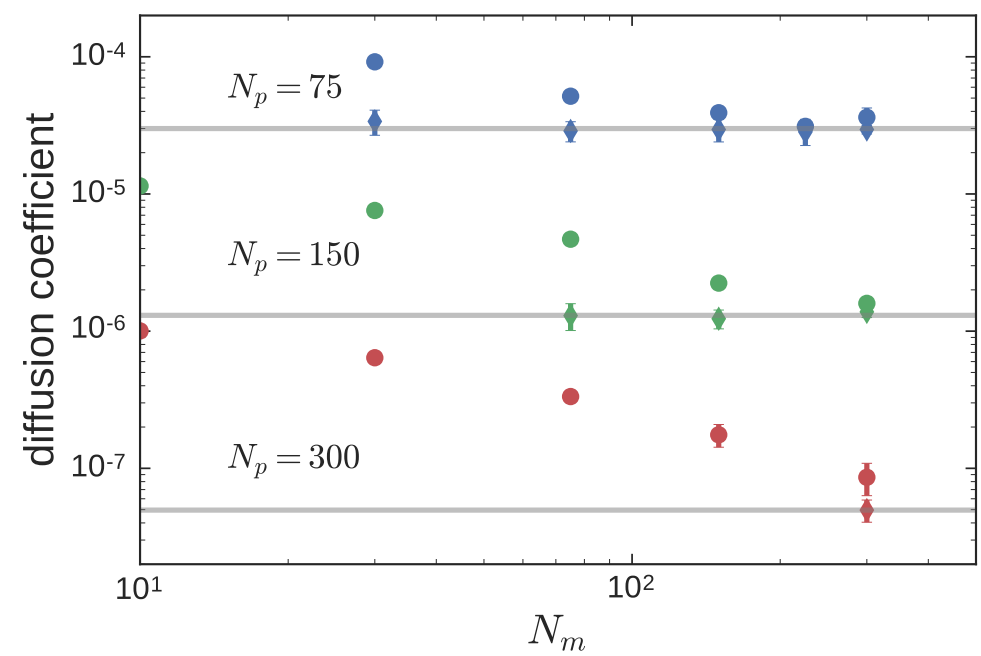

Figure 6: Diffusion coefficient of probes (circles) with $N_{p}=75$ (blue), 150 (green), and $N_{p}=300$ (red) in matrices with $N_{m}=10-300$. Simulations with clamped matrix chains are depicted by diamonds; horizontal gray lines depict the mean values, $\left\langle\hat{D}\left(N_{p}\right)\right\rangle$. For clarity, all the diffusion coefficients corresponding to $N_{p}=150$ and 300 are shifted downwards by a factor of 5 and 25 , respectively.

$N_{p}=75$ and 150, CM simulations were carried out at five and three different values of $N_{m}$, respectively. At a particular choice of $N_{p}$ and $N_{m}$, the effect of fixing matrix chain ends is reflected in the difference between the probe and CM diffusivities. Unlike $D, \hat{D}$ appears to be relatively independent of $N_{m}$.

\subsubsection{Estimating Tracer Diffusivity from CM Simulations}

Due to the immobilization constraint, we expect $\hat{D} \leq D$ for a given $N_{p}$ and $N_{m}$. The difference between $\hat{D}$ and $D$ is large at small $N_{m}$. As $N_{m}$ increases, $D$ converges to $\hat{D}$ (fig. 6). Furthermore, $\hat{D}$ for a particular $N_{p}$ is insensitive to $N_{m}$, for moderately and well-entangled matrix chains $\left(N_{m} \gtrsim 75-100\right)$. We assume that this insensitivity can be extrapolated to longer matrix chains, $N_{m} \gg N_{p}$. These two observations: the convergence of $D$ to $\hat{D}$ from "above" as $N_{m}$ increases, and the independence of $\hat{D}$ and $N_{m}$, necessarily imply that tracer diffusivity $D_{\infty}$ may be directly estimated from $\hat{D}$.

To improve statistics, we average $\hat{D}\left(N_{p}, N_{m}\right)$ over different values of $N_{m}$ (when available) to calculate the mean $\left\langle\hat{D}\left(N_{p}\right)\right\rangle$. In figure $6,\left\langle\hat{D}\left(N_{p}\right)\right\rangle$ is shown by gray lines 
that pass through the CM diffusivities. From the argument above, we claim that this quantity provides a good estimate of the tracer diffusivity, i.e. $D_{\infty}\left(N_{p}\right) \approx\left\langle\hat{D}\left(N_{p}\right)\right\rangle$ (see SI section 4).

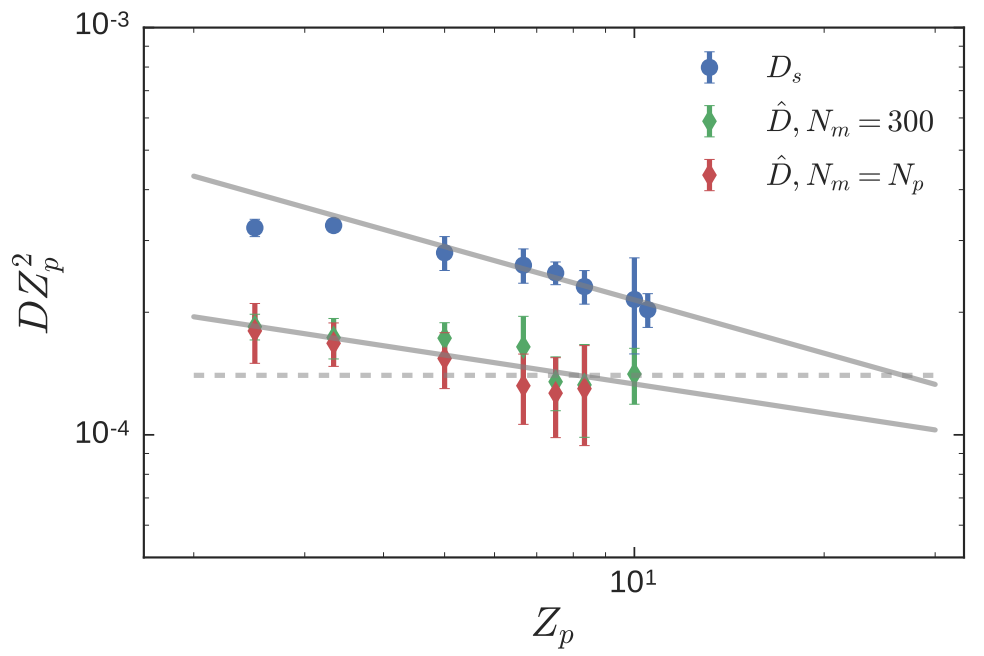

Figure 7: BFM Simulations: Blue circles depict $D_{s}$ scaled by $Z_{p}^{2}$, and best-fit line (gray) has a slope $Z_{p}^{-0.43}$. Diamonds depict tracer diffusivity (scaled by $Z_{p}^{2}$ ) obtained from CM simulations with matrix chains of $N_{m}=300$ (green) and $N_{m}=N_{p}$ (red). The downward sloping gray line passing through these points corresponds to $Z_{p}^{-0.24}$. The horizontal dashed line corresponds to the pure reptation limit for which $\hat{D} Z_{p}^{2}$ is constant.

\subsection{Self-Diffusivity and Tracer Diffusivity}

Tracer diffusivity $D_{\infty}$ is estimated from the BFM simulations using the average $\langle\hat{D}\rangle$. Including probe chains longer than $N \geq 100$, we obtain a best-fit $D_{\infty}=(2.30 \pm 0.11) \times$ $10^{-4} Z^{-2.24 \pm 0.03}$. This is in contrast with previously shown (fig. 4) results for selfdiffusivity $D_{s}$, for which the corresponding best-fit was $D_{s} \approx 5.84 \times 10^{-4} Z^{-2.43}$. Not only is $D_{\infty}<D_{s}$ over the range of molecular weights investigated as expected, it also has a weaker dependence on chain length. While $x_{s}=2.43 \pm 0.07$ is consistent with the empirical observation of $x_{s}=2.4 \pm 0.2, x_{\infty}=2.24 \pm 0.03$ obtained from BFM is somewhat stronger than $x_{\infty}=2.0 \pm 0.1$ reported in experiments. ${ }^{12}$

The difference in $x_{s}$ and $x_{\infty}$ can be emphasized by multiplying $D_{s}$ and $D_{\infty}$ with 
$Z_{p}^{2}$, so that deviations from a slope of $Z_{p}^{-2}$ become salient. Figure 7 includes results of $\mathrm{CM}$ simulations at two different values of $Z_{m}: Z_{m}=Z_{p}$ similar to the systems used to extract $D_{s}$ but with clamped matrix chain ends, and $Z_{m}=10$, which is the longest matrix chain explored with BFM in this study. As anticipated, both these CM calculations lead to similar estimates of $D_{\infty}$. The solid lines in the figure 7 have slopes of -0.43 and -0.24 , reflecting $x_{s}=2.43$ and $x_{\infty}=2.24$. The dashed horizontal line depicts the pure reptation limit. The best-fit through the self-diffusion data, and the dashed line intersect at $Z_{1}^{*} \approx 25-30$. Instead, if we use best-fits shown by the two solid gray lines, we obtain $Z_{1}^{*} \approx 100$. This demonstrates the sensitivity of $Z_{1}^{*}$ to $x_{s}$ and $x_{\infty}$. In any case, modeling polymers of this size $\left(Z_{p} \gtrsim Z_{1}^{*}\right)$ by brute force simulations is currently beyond the reach of the BFM.

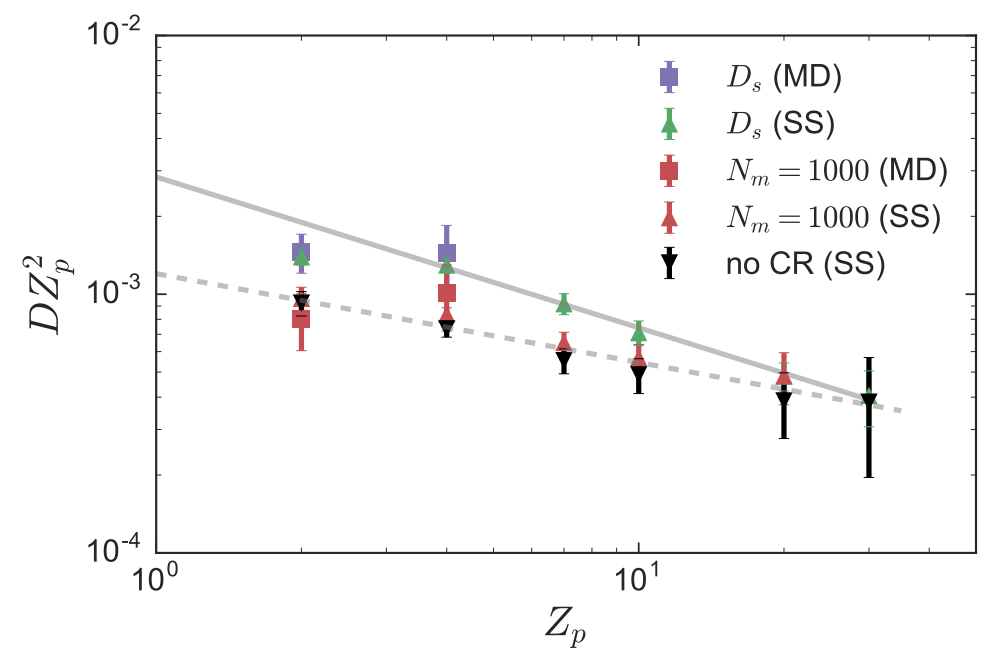

Figure 8: MD and SS Simulations: Purple squares (MD) and green triangles (SS) depict $D_{s}$ scaled by $Z_{p}^{2}$. The best-fit line (gray) has a slope $Z_{p}^{-0.59}$. Red symbols correspond to scaled probe diffusivities obtained from MD (square) and SS (triangles) simulations of binary blends with $N_{m}=1000$. Black inverted triangles correspond to scaled probe diffusion coefficients obtained from SS simulations with CR switched off. The corresponding dashed line has a slope $Z_{p}^{-0.34}$.

Figure 8 for SS and MD simulations is analogous to figure 7. It reproduces the scaled self-diffusion coefficients previously shown in fig. 4 for MD (purple squares) and SS (green triangles) simulations. The gray line passing through the points corresponds to 
the best fit with slope $x_{s}=2.59$. Red symbols depict probe diffusivity $D$ (scaled by $Z_{p}^{2}$ ) in a matrix with $N_{m}=1000$, using MD (squares) and SS simulations (triangles). As expected, $D<D_{s}$ at a given $Z_{p}$, because the matrix chains are longer than the probes, $N_{m} \geq N_{p}$. A qualitatively similar trend is observed for BFM simulations, and expected from eqns 1 and 2. For $N_{p} \leq 200$, where MD is feasible, the agreement with probe diffusivity estimated from SS simulations is good within error bars, which essentially validates the mapping of the SS model parameters to those of MD. For $Z_{p} \geq 3$, the bestfit through the probe diffusivities corresponds to $D=(1.23 \pm 0.11) \times 10^{-3} Z^{-2.32 \pm 0.04}$. The decrease in the magnitude of the probe diffusivity relative to the self-diffusivity $\left(D / D_{s}\right)$ is less pronounced in SS simulations compared with BFM. For example, at $Z_{p} \approx 8(N=250$ in $\mathrm{BFM}$ and $N=500$ in $\mathrm{MD}$ units $), D_{\infty} / D_{s} \approx 0.57$ in the BFM, while $D / D_{s} \approx 0.70$ in the SS model.

The diffusivity $\tilde{D}$ obtained from SS simulations with CR switched off is shown by black triangles. In table 7 of SI, $\tilde{D}$ at a given $Z_{p}$ is shown to be slightly smaller (by $\approx 20 \%$ ) than its counterpart obtained from binary blends with the same $Z_{p}$. For $Z_{p} \geq 4\left(N_{p} \geq 200\right.$ in MD units), the best-fit through the tracer diffusivities is $D_{\infty} \equiv$ $\tilde{D}=(1.09 \pm 0.21) \times 10^{-3} Z^{-2.33 \pm 0.08}$ for the range of $Z_{p}$ studied. For improved statistics, we can combine the probe diffusivity results obtained in binary blends with $\tilde{D}$. This yields $x_{\infty}=2.34 \pm 0.04$ which is shown in figure 8 as the best-fit line (dashed gray line).

At $Z=30$, the measured values of $D_{s}$ and $\tilde{D}$ actually coincide, as do extrapolations of the best fits for the self and tracer diffusion coefficients. This implies $Z_{1}^{*} \approx 30$ in the SS simulations, although further simulations with larger $Z_{p}$ are needed to draw a solid conclusion. This $Z_{1}^{*}$ value is consistent with the theoretical prediction ${ }^{13}$ and some analysis of experiments. ${ }^{12}$

Thus, we can answer the two questions we started out with. "Is CR responsible for the experimentally observed difference between $x_{s}$ and $x_{\infty}$ ?" The answer from both BFM and SS simulations is categorically affirmative. The ambiguity around the relative magnitudes of the two exponents in some older simulations is resolved. We 
demonstrate that two independent methods (BFM and SS) used to infer both $x_{s}$ and $x_{\infty}$ using the same or similar simulation protocol agree that $x_{s}>x_{\infty}$. This brings molecular simulations qualitatively in line with theory and experiments, and eliminate a source of uncertainty in the literature for $Z<Z_{1}^{*}$. "Can CLF lead to deviation of $x_{\infty}$ from pure reptation behavior (i. e., $x_{\infty}>2$ )?" Again, the answer from both BFM and SS simulations appears to be affirmative. This is discussed in greater detail in the next section.

\subsection{Contributions of Constraint Release and Contour Length Fluctuations}

The similarities and differences between the $x_{s}$ and $x_{\infty}$ obtained from experiments, theory, and simulations warrant further discussion. The magnitudes of exponents $\left(x_{s}\right.$ and $\left.x_{\infty}\right)$, and the critical entanglement numbers $\left(Z_{1}^{*}\right.$ and $\left.Z_{2}^{*}\right)$ are reported in table 1 . In this work, simulations are confined to $Z_{p} \lesssim Z_{1}^{*}$ due to computational cost. This means that the transition to pure reptation scaling marked by $Z_{2}^{*}$ is not directly addressed. Similarly, as pointed out earlier, experiments suggest $Z_{1}^{*}=Z_{2}^{*} \cdot{ }^{12}$

In table $1, x_{s}$ is remarkably consistent across the different methods within the window of variation. It should be pointed out that for $Z<Z_{1}^{*}$, the theoretical model does not exhibit a constant exponent; ${ }^{13}$ instead $x_{s}$ decreases monotonically with increasing $Z$. The value reported in the table is the best-fit for $5 \leq Z \leq Z_{1}^{*}$. For lightly entangled polymers $(Z<5)$, the theoretical model may not be reliable and predicts a significantly larger $x_{s}$.

From table 1 , values of $x_{\infty} \approx 2.25-2.35$ obtained from theory, BFM, and SS simulations are also reasonably consistent with each other. However, they are somewhat larger than the $x_{\infty}$ obtained from experiments. In particular, $x_{\infty}>2$, which suggests the existence of an additional relaxation mechanism other than pure reptation in the simulated chains. Since CR is ruled out in the tracer diffusivity measurements, the only remaining effect is CLF. 
Table 1: Summary of the exponents for tracer and self-diffusivity using molecular simulations (BFM and SS) compared to theory ${ }^{13}$ and experiments. ${ }^{12}{ }^{a}$ In theoretical predictions, ${ }^{13}$ the value of $x_{s}$ decreases with $Z$ monotonically; the best-fit of $x_{s}$ for the range of $5 \leq Z \leq Z_{1}^{*}$ is reported here.

\begin{tabular}{c|cc|cc}
\hline & $x_{s}$ & $x_{\infty}$ & $Z_{1}^{*}$ & $Z_{2}^{*}$ \\
\hline experiments & $2.4 \pm 0.2$ & $2.0 \pm 0.1$ & $\sim 20-100$ & $Z_{1}^{*}$ \\
theory & $2.5 \pm 0.1^{a}$ & 2.25 & $\sim 20$ & $200-1000$ \\
BFM & $2.43 \pm 0.07$ & $2.24 \pm 0.03$ & $\sim 25-100$ & - \\
SS & $2.59 \pm 0.05$ & $2.34 \pm 0.04$ & $\sim 30$ & - \\
\hline
\end{tabular}

Following the theoretical model of Frischknecht and Milner, CLF can lead to an enhancement of the diffusion coefficient $D_{\infty}$ by a factor of $\left(1-s_{d}\right)^{-1}$ where $0 \leq s_{d} \leq 1$ is the so-called fractional arm retraction distance at the reptation time $\tau_{d}$ of the linear chain. ${ }^{13}$ The dependence of $s_{d}$ on $Z_{p}$ can be calculated from the crossover of the arm retraction time by treating the chain as a two-arm star. For probe chains diffusing in a permanent entanglement network, the tracer diffusion coefficient is predicted to scale as $Z_{p}^{-2.25}$, which is reasonably consistent with results of both sets of molecular simulations. This agreement over the range of $1 \ll Z_{p} \lesssim Z_{1}^{*}$ supports the theoretical suggestion that CLF alone can result in faster diffusion of probe chains than pure reptation behavior. Indirectly, these simulations also support the idea of an intermediate molecular weight regime $Z_{1}^{*}<Z<Z_{2}^{*}$ where $D_{s} \approx D_{\infty}$ and both $\mathrm{CLF}$ and reptation are active.

It remains unclear why the CLF effect on $x_{\infty}$ is not (sufficiently) reflected in the experimental data, ${ }^{12}$ especially for weakly entangled probe chains with $Z_{p} \sim \mathcal{O}(1)$, where CLF ought to dominate chain dynamics. ${ }^{68}$ On the other hand, the probe chain lengths studied in the SS simulations are not long enough to examine the validity of the theoretical hypothesis that CR effect is negligible in determining the self-diffusion coefficient for probe chains with $Z_{p}>Z_{1}^{*} .{ }^{13}$

Interestingly, the difference $\left|x_{s}-x_{\infty}\right|$ for experiments and simulations is consistent within reported uncertainties: it is $0.40 \pm 0.22$ for experiments, $0.19 \pm 0.08$ for BFM, and $0.25 \pm 0.07$ for SS simulations. The location of the first critical entanglement number $Z_{1}^{*}$ is sensitive to this difference, and varies inversely with it. From table 1 , all 
the methods suggest that $Z_{1}^{*}$ lies within a somewhat broad range of $\sim 20-100$.

\section{Summary and Conclusions}

We used two different molecular simulation methods, BFM and SS model calibrated with MD, to study the difference in the molecular weight dependence of tracer diffusivity $\left(D_{\infty} \sim Z^{-x_{\infty}}\right)$, and self-diffusion coefficient $\left(D_{s} \sim Z^{-x_{s}}\right)$. SS simulations are more coarse-grained, and allow us to explore a wider range of probe molecular weights (up to the first critical entanglement number $Z_{1}^{*} \approx 30$ ).

Estimating $x_{\infty}$ using brute-force MD or BFM simulations is ill-advised, because it involves large simulation boxes and long trajectories. Therefore, we presented and validated a new method to estimate $D_{\infty}$ with BFM simulations, in which the ends of the matrix chains are immobilized (CM simulation). Constraint release can be turned off in SS model, which allows us to compute $x_{\infty}$ directly.

One of the primary goals of this study was to resolve disagreement between experiments and theory which claimed $x_{s}>x_{\infty}$, over a wide intermediate molecular weight range $\left(1 \ll Z<Z_{1}^{*}\right)$, and older simulations which sometimes claimed the opposite, $x_{\infty}>x_{s}$. The calculations reported in this work unambiguously demonstrate that $x_{s}>x_{\infty}$ in molecular simulations, bringing them in line with expectations from theory and experiments. Furthermore, the difference between $x_{s}$ and $x_{\infty}$ can be attributed mainly to CR effects.

Despite qualitative agreement between the methods, important quantitative differences persist. For $Z_{p} \lesssim Z_{1}^{*}$, theory predicts a much stronger dependence of $D_{s}$ on molecular weight than experiments or simulations. The situation is reversed for tracer diffusion, where $x_{\infty}$ obtained from simulations and theory is larger than that obtained from experiments. The higher value of $x_{\infty}$ strongly suggests that the influence of CLF may be underrated in the analysis of experimental data. Perhaps, the experimental data on $D_{\infty}$ requires re-examination, in particular for $Z_{p} \leq 10$, where the CLF effects are expected to play important role. 


\section{Acknowledgement}

This material is based partially upon work partially supported by the National Science Foundation under Grant No. DMR 1727870 (SS). Z. Wang acknowledges the support of Engineering and Physical Sciences Research Council (EPSRC) Grant No. EP/L020599/1. The authors would like to thank S. Q Wang, A. E. Likhtman, H. X. Guo, and R. G. Larson for helpful discussions.

\section{Supporting Information}

Supporting information contains: (i) summary of systems investigated, (ii) tabulated self and tracer diffusion data, (iii) comparison with MCSS and PCN simulations, and (iv) tracer diffusivity from clamped matrix simulations

\section{References}

(1) Green, P. F.; Kramer, E. J. Matrix effects on the diffusion of long polymer chains. Macromolecules 1986, 19, 1108-1114.

(2) Graessley, W. W. Entangled linear, branched and network polymer systems Molecular theories. Advan. Polym. Sci. 1982, 47, 67-117.

(3) Klein, J. Dynamics of entangled linear, branched, and cyclic polymers. Macromolecules 1986, 19, 105-118.

(4) Antonietti, M.; Coutandin, J.; Sillescu, H. Diffusion of linear polystyrene molecules in matrixes of different molecular weights. Macromolecules 1986, 19, 793-798.

(5) Watanabe, H.; Kotaka, T. Viscoelastic and diffusion properties of binary blends of monodisperse polystyrenes. Macromolecules 1987, 20, 530-535. 
(6) Nemoto, N.; Kojima, T.; Inoue, T.; Kishine, M.; Hirayama, T.; Kurata, M. Self diffusion of polymers in the concentrated regime. Part 2. Self diffusion and tracerdiffusion coefficient and viscosity of concentrated solutions of linear polystyrenes in dibutyl phthalate. Macromolecules 1989, 22, 3793-3798.

(7) Gent, A. N.; Kaang, S. Y. Diffusion of linear polyisoprene molecules into polyisoprene networks. J. Polym. Sci. B: Polym. Phys. 1989, 27, 893-911.

(8) Seggern, J. V.; Klotz, S.; Cantow, H. J. Reptation and constraint release in linear polymer melts: an experimental study. Macromolecules 1991, 24, 3300-3303.

(9) Wang, S.; von Meerwall, E. D.; Wang, S.-Q.; Halasa, A.; Hsu, W.-L.; Zhou, J. P.; Quirk, R. P. Diffusion and rheology of binary polymer mixtures. Macromolecules 2004, 37, 1641-1651.

(10) Lodge, T. P. Reconciliation of the molecular weight dependence of diffusion and viscosity in entangled polymers. Phys. Rev. Lett. 1999, 83, 3218-3221.

(11) Tao, H.; Lodge, T. P.; von Meerwall, E. D. Diffusivity and viscosity of concentrated hydrogenated polybutadiene solutions. Macromolecules 2000, 33, 17471758.

(12) Wang, S.-Q. Chain dynamics in entangled polymers: Diffusion versus rheology and their comparison. J. Polym. Sci. B: Polym.Phys. 2003, 41, 1589-1604.

(13) Frischknecht, A. L.; Milner, S. T. Diffusion with contour length fluctuations in linear polymer melts. Macromolecules 2000, 33, 5273-5277.

(14) Liu, C. Y.; Keunings, R.; Bailly, C. Do deviations from reptation scaling of entangled polymer melts result from single- or many-chain effects? Phys. Rev. Lett. 2006, 97, 246001.

(15) Doi, M. Explanation for the 3. 4-power law for viscosity of polymeric liquids on the basis of the tube model. J. Polym. Sci. B: Polym. Phys. 1983, 21, 667-684. 
(16) Likhtman, A. E.; McLeish, T. C. B. Quantitative theory for linear dynamics of linear entangled polymers. Macromolecules 2002, 35, 6332-6343.

(17) Nair, D. M.; Schieber, J. D. Linear viscoelastic predictions of a consistently unconstrained Brownian slip-link model. Macromolecules 2006, 39, 3386-3397.

(18) Kremer, K.; Grest, G. S. Dynamics of entangled linear polymer melts: A molecular-dynamics simulation. J. Chem. Phys. 1990, 92, 5057-5086.

(19) Paul, W.; Binder, K.; Heermann, D. W.; Kremer, K. Crossover scaling in semidilute polymer solutions: A Monte Carlo test. J. Phys. II France 1991, 1, 37-60.

(20) Shaffer, J. S. Effects of chain topology on polymer dynamics - Bulk melts. J. Chem. Phys. 1994, 101, 4205-4213.

(21) Smith, S. W.; Hall, C. K.; Freeman, B. D. Molecular dynamics study of entangled hardchain fluids. J. Chem. Phys. 1996, 104, 5616-5637.

(22) Binder, K.; Paul, W. Monte Carlo simulations of polymer dynamics: Recent advances. J. Polym. Sci. Polym. Phys. 1997, 35, 1-31.

(23) Deutsch, J. M.; Madden, T. L. The diffusion coefficient of a reptating polymer. J. Chem. Phys. 1989, 91, 3252-3257.

(24) Baumgärtner, A.; Ebert, U.; Schäfer, L. Segment motion in the reptation model of polymer dynamics. II. Simulations. J, Stat. Phys. 1998, 90, 1375-1400.

(25) Reiter, J. A random walk chain reptating in a network of obstacles: Monte Carlo study of diffusion and decay of correlations and a comparison with the rouse and reptation models. J. Chem. Phys. 1991, 94, 3222-3228.

(26) Barkema, G. T.; Krenzlin, H. M. Long-time dynamics of de gennes model for reptation. J. Chem. Phys. 1998, 109, 6486-6489. 
(27) Kreer, T.; Baschnagel, J.; Müller, M.; Binder, K. Monte Carlo simulation of long chain polymer melts: crossover from Rouse to reptation dynamics. Macromolecules 2001, 34, 1105-1117.

(28) Harmandaris, V. A.; Mavrantzas, V. G.; Theodorou, D. N.; Krger, M.; Ramrez, J.; ttinger, H. C.; Vlassopoulos, D. Crossover from the rouse to the entangled polymer melt regime: Signals from long, detailed atomistic molecular dynamics simulations, supported by rheological experiments. Macromolecules 2003, 36, $1376-1387$.

(29) Harmandaris, V. A.; Kremer, K. Dynamics of polystyrene melts through hierarchical multiscale simulations. Macromolecules 2009, 42, 791-802.

(30) Takahashi, K. Z.; Nishimura, R.; Yasuoka, K.; Masubuchi, Y. Molecular dynamics simulations for resolving scaling laws of polyethylene melts. Polymers 2017, 9, 24.

(31) Masubuchi, Y.; Takimoto, J.-I.; Koyama, K.; Ianniruberto, G.; Marrucci, G.; Greco, F. Brownian simulations of a network of reptating primitive chains. $J$. Chem. Phys. 2001, 115, 4387-4394.

(32) Hagita, K.; Takano, H. Self-diffusion of a polymer chain in a melt. J. Phys. Soc. Jpn. 2003, 72, 1824-1827.

(33) Likhtman, A. E. Single-chain slip-link model of entangled polymers: Simultaneous description of neutron spin-echo, rheology, and diffusion. Macromolecules 2005, 38, 6128-6139.

(34) Karatrantos, A.; Composto, R. J.; Winey, K. I.; Kröger, M.; Clarke, N. Modeling of Entangled Polymer Diffusion in Melts and Nanocomposites: A Review. Polymers 2019, 11, 876 .

(35) Shanbhag, S. Unusual dynamics of ring probes in linear matrices. Journal of Polymer Science Part B: Polymer Physics 2017, 55, 169-177. 
(36) Masubuchi, Y.; Uneyama, T. Comparison among multi-chain models for entangled polymer dynamics. Soft Matter 2018, 14, 5986-5994.

(37) Baschnagel, J.; Paul, W.; Tries, V.; Binder, K. Statics and dynamics of bidisperse polymer melts: A Monte Carlo study of the bond-fluctuation model. Macromolecules 1998, 31, 3856-3867.

(38) Barsky, S. Molecular dynamics study of diffusion in bidisperse polymer melts. $J$. Chem. Phys. 2000, 112, 3450-3456.

(39) Lin, H.; Mattice, W. L.; von Meerwall, E. D. Chain dynamics of bidisperse polyethylene melts: A Monte Carlo study on a high-coordination lattice. Macromolecules 2007, 40, 959-966.

(40) Picu, R. C.; Rakshit, A. Coarse grained model of diffusion in entangled bidisperse polymer melts. J. Chem. Phys. 2007, 127.

(41) Wang, Z.; Larson, R. G. Constraint release in entangled binary blends of linear polymers: A molecular dynamics study. Macromolecules 2008, 41, 4945-4960.

(42) Wang, Z.; Likhtman, A. E.; Larson, R. G. Segmental dynamics in entangled linear polymer melts. Macromolecules 2012, 45, 3557-3570.

(43) Sukumaran, S. K.; Likhtman, A. E. Modeling entangled dynamics: comparison between stochastic single-chain and multichain models. Macromolecules 2009, 42, 4300-4309.

(44) Auhl, R.; Everaers, R.; Grest, G. S.; Kremer, K.; Plimpton, S. J. Equilibration of long chain polymer melts in computer simulations. J. Chem. Phys. 2003, 119, $12718-12728$.

(45) Takahashi, K. Z.; Yamato, N.; Yasuoka, K.; Masubuchi, Y. Critical test of beadspring model to resolve the scaling laws of polymer melts: a molecular dynamics study. Molecular Simulation 2017, 43, 1196-1201. 
(46) Pütz, M.,; Kremer, K.,; Grest, G. S., What is the entanglement length in a polymer melt? Europhys. Lett. 2000, 49, 735-741.

(47) Everaers, R.; Sukumaran, S. K.; Grest, G. S.; Svaneborg, C.; Sivasubramanian, A.; Kremer, K. Rheology and microscopic topology of entangled polymeric liquids. Science 2004, 303, 823-826.

(48) Ramirez, J.; Sukumaran, S. K.; Likhtman, A. E. Hierarchical modeling of entangled polymers. Macromol. Symp. 2007, 252, 119-129.

(49) Hoy, R. S.; Foteinopoulou, K.; Kröger, M. Topological analysis of polymeric melts: chain-length effects and fast-converging estimators for entanglement length. Phys. Rev. E 2009, 80, 031803.

(50) Hou, J.-X.; Svaneborg, C.; Everaers, R.; Grest, G. S. Stress Relaxation in Entangled Polymer Melts. Phys. Rev. Lett. 2010, 105, 068301.

(51) Everaers, R. Topological versus rheological entanglement length in primitive-path analysis protocols, tube models, and slip-link models. Phys. Rev. E 2012, 86, 022801.

(52) Zhou, Q.; Larson, R. G. Direct molecular dynamics simulation of branch point motion in asymmetric star polymer melts. Macromolecules 2007, 40, 3443-3449.

(53) Carmesin, I.; Kremer, K. The bond fluctuation method - a new effective algorithm for the dynamics of polymers in all spatial dimensions. Macromolecules 1988, 21, $2819-2823$.

(54) Shanbhag, S.; Larson, R. G. Chain retraction potential in a fixed entanglement network. Phys. Rev. Lett. 2005, 94(7), 076001.

(55) Subramanian, G.; Shanbhag, S. On the relationship between two popular lattice models for polymer melts. J. Chem. Phys. 2008, 129, 144904. 
(56) Shanbhag, S.; Larson, R. G. Identification of topological constraints in entangled polymer melts using the bond-fluctuation model. Macromolecules 2006, 39, 24132417.

(57) Shanbhag, S.; Kroger, M. Primitive path networks generated using annealing and geometrical methods: Insights into differences. Macromolecules 2007, 40, 28972903.

(58) Iyer, B. V. S.; Shanbhag, S.; Juvekar, V. A.; Lele, A. K. Self-diffusion coefficient of ring polymers in semidilute solution. J. Polym. Sci. B: Polym. Phys. 2008, 46, $2370-2379$.

(59) Subramanian, G.; Shanbhag, S. Self-diffusion in binary blends of cyclic and linear polymers. Macromolecules 2008, 41, 7239-7242.

(60) Shanbhag, S. Estimating self-diffusion in polymer melts: how long is a long enough molecular simulation? Mol. Simul. 2016, 42, 162-172.

(61) Crysup, B.; Shanbhag, S. What happens when threading is suppressed in blends of ring and linear polymers? Polymers 2016, 8 .

(62) Shanbhag, S. Extraction of self-diffusivity in systems with nondiffusive short-time behavior. Phys. Rev. E 2013, 88, 042816.

(63) Cao, J.; Wang, Z. Microscopic picture of constraint release effects in entangled star polymer melts. Macromolecules 2016, 49, 5677-5691.

(64) Zhu, J.; Likhtman, A. E.; Wang, Z. Arm retraction dynamics of entangled star polymers: A forward flux sampling method study. J. Chem. Phys. 2017, 147, 044907.

(65) Chapman, C. D.; Shanbhag, S.; Smith, D. E.; Robertson-Anderson, R. M. Complex effects of molecular topology on diffusion in entangled biopolymer blends. Soft Matter 2012, 8, 9177-9182. 
(66) Henke, S. F.; Shanbhag, S. Self-diffusion in asymmetric ring-linear blends. React. Func. Polym. 2014, 80, $57-60$.

(67) Shivokhin, M. E.; Read, D. J.; Kouloumasis, D.; Kocen, R.; Zhuge, F.; Bailly, C.; Hadjichristidis, N.; Likhtman, A. E. Understanding effect of constraint release environment on end-to-end vector relaxation of linear polymer chains. Macromolecules 2017, 50, 4501-4523.

(68) Nedelcu, S.; Sommer, J.-U. Single-chain dynamics in frozen polymer networks. Rheol. Acta 2010, 49, 485-494. 